\title{
Signal Processing Technique for Time Domain Measurement of S-Parameters
}

\author{
Isabela M. Nobre, Julio L. Nicolini, Joaquim D. Garcia, Marbey Mosso \\ Centre of Studies in Telecomunications, Department of Electrical Engineering, Pontifical Catholic University of \\ Rio de Janeiro, Rio de Janeiro, Brazil \\ belanobre@hotmail.com,juliolnicolini@gmail.com, joaquimdgarcia@gmail.com, marbeyg@gmail.com
}

\begin{abstract}
In this paper, a simple and accurate technique for the measurement of the S-Parameters of a two-port network from time domain data is presented, based on Fourier theory and techniques. This method is proposed to circumvent the need of a network analyser when studying an unknown device, as no additional measurements other than the time domain data are required. An excellent correspondence between this technique's results and regular network analyser measurements is achieved for the entire operational band of the devices tested.
\end{abstract}

Index Terms - Instrumentation, Microwave, Signal Processing, Time Domain Reflection.

\section{INTRODUCTION}

In the microwave community, scattering parameters (S-parameters) are frequently used to characterize a device under test (DUT). It has been shown that power waves and the corresponding scattering matrix give a clear understanding of the power relations between circuit elements connected through a multiport network [1]. Microwave measurement techniques for component and system characterization and modeling can be categorized as Frequency Domain Measurements Techniques (FDMT) and Time Domain Measurements Techniques (TDMT) [2]. In this paper, the focus will be in extracting frequency information from a time domain method. In this technique, a network can be analyzed in two ways: the Time Domain Reflection (TDR) and the Time Domain Transmission (TDT) methods. Due to the lower costs of TDR and TDT instruments, the acquisition of frequencydomain parameters by using time-domain data is a growing alternative to traditional frequencydomain analysis [3].

In this paper it is described a method of extracting the S-parameters of a device under test by the use of TDR and TDT techniques. The Discrete Fourier Transform is applied to the incident and the reflected/transmitted signals. The reflection/transmission coefficients are obtained by the division of the transformed reflected/transmitted signals to the transformed incident signal. All the processing is done by the use of the software MATLAB.

\section{TIME DOMAIN MEASUREMENTS IN MICROWAVE FREQUENCIES}

TDR is a measurement technique in which a pulse of energy is transmitted through a cable. When 
that pulse reaches the end of the cable, or a fault along the cable, part of it is reflected back. The measurement is made by launching a step into the DUT and observing the response in time. The operation is similar for the TDT technique, but with the transmitted pulse. TDR can be used to determine the return loss, the standing wave ratio, the reflection coefficient, and the scattering parameters $\mathrm{S}_{11}$ and $\mathrm{S}_{22}$ of a DUT. On the other hand, TDT can be used to determine the propagation time, the length, the gain or loss, the crosstalk, the transmission coefficient, and the scattering parameters $\mathrm{S}_{21}$ and $\mathrm{S}_{12}$ of a two-port DUT [2].

The time domain reflectometry theory is based on the use of a step generator and an oscilloscope. The step is launched into the transmission line under test. The oscilloscope monitors the incident and reflected voltage at a particular point of the line. In the display, it is possible to have information about the impedance of the line, along with the position and nature of each discontinuity [4].

Noise is an important factor in data analysis in laboratory measurements. All laboratory procedures are subject to a variety of noise sources, therefore a processing technique is used in the results of TDR and TDT measurements realized in this work to minimize noise. Many filters can be used to smooth the time domain measurements, such as the 11 trend filter [5], the Savintsky Golay filter [6] and the Hodrick Prescott filter [7]. The Hodrick Prescott filter was initially used on econometrics, however as it has smoother results it has also found applications in chemistry [8] and control theory [9]. In this paper we use the Hodrick Prescott filter to smooth and remove part of the noise of the time domain signal.

\section{MATHEMATICAL MODEL}

\section{A. Electromagnetic Theory}

If the load connected to the cable has different impedance, as shown in figure 1, Maxwell's equations are only satisfied if a second wave is originated at the load and propagates back towards the source. The ratio of the reflected wave to the incident wave is defined as the reflection coefficient [10]:

$$
\Gamma=\frac{E_{r}}{E_{i}}=\frac{Z_{L}-Z_{0}}{Z_{L}+Z_{0}}
$$

If a mismatch exists at the load, the reflected wave will be added algebraically to the incident wave at the oscilloscope display [4]. Since it is separated in time from the incident wave, the reflected wave can be identified. The shape is also valuable to reveal the nature and the magnitude of the mismatch.

The same is valid for the transmitted wave, which exists to ensure Maxwell's equations on the other side of the discontinuity. The transmission coefficient can be defined as [10]:

$$
T=\frac{E_{t}}{E_{i}}=\frac{2 Z_{L}}{Z_{L}+Z_{0}}
$$

By this method, it is obtained the responses in time to the step generator (transmitted and reflected responses). 


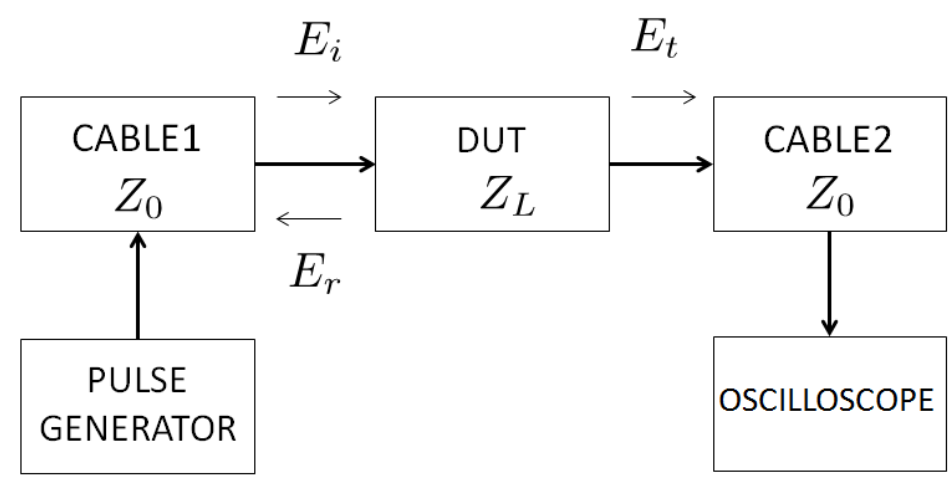

Fig. 1. Circuit block diagram.

\section{B. Data Processing}

To extract the continuous signals for future computer analysis, it is required to perform a sampling. A continuous signal sampled on a grid finer than the Nyquist interval can be, in principle, perfectly reconstructed via interpolation, since the sampling does not compromise the information content of the signal.

The Nyquist interval is the upper bound on the sampling interval of a discretized signal in the time domain such that the sample contains all the available frequency information of the signal.

The Nyquist-Shannon sampling theorem states [11]:

"If a function $\mathrm{x}(\mathrm{t})$ contains no frequencies higher than B Hertz, it is completely determined by giving its ordinates at a series of points spaced 1/2B seconds apart"

The threshold $2 \mathrm{~B}$ is called the Nyquist rate and $1 / 2 \mathrm{~B}$ is the Nyquist interval.

By the use of the proper sampling rate, we obtain the output and input signals in the discrete time domain. They are related to the discrete time impulse response by the following:

$$
y[k]=h[k] * u[k]
$$

Where $y[k]$ is the sampled output signal, $u[k]$ the sampled input signal, $h[k]$ the discrete- time impulse response, and the “*” signal denotes the convolution sum operation [12], given by:

$$
y[k]=\sum_{i=-\infty}^{\infty} u[i] h[k-i]
$$

The discrete-time impulse response is related to the scattering parameters. It is also more directly related to the discrete transfer function of the system through the use of the Discrete Fourier Transform (DFT). The DFT of a signal $\mathrm{x}[\mathrm{k}]$ can be defined as [13]:

$$
X(f)=\frac{1}{N} \sum_{k=0}^{N-1} x[k] e^{\frac{i 2 \pi f k}{N}}
$$

Where $\mathrm{N}$ is the number of samples. 
One of the properties of this transformation is that the convolution sum of two signals in the time domain becomes a simple product of the transformed signals in the domain of the transformation (named frequency domain) [12]. By this property, equation 4 becomes:

$$
\hat{Y}(f)=\hat{H}(f) \cdot \hat{U}(f)
$$

Where $\hat{Y}$ and $\hat{U}$ are, respectively, the DFT of the time-domain signals $y[k]$ and $\mathrm{u}[\mathrm{k}]$, and $\mathrm{H}$ is the discrete transfer function of the system, or the discrete frequency response. Thus, it is easier to obtain $\hat{\mathrm{H}}$ than $\mathrm{h}[\mathrm{k}]$ by the use of the previous equation, which rearranged, becomes:

$$
\hat{H}(f)=\frac{\hat{Y}(f)}{\hat{U}(f)}
$$

The transformation to the frequency domain of $y[k]$ and $u[k]$ is done by the Chirp Z-Transform algorithm (CZT). The reference [14] discusses this algorithm in details. In this reference, it is stated that the CZT is

"A computational algorithm for numerically evaluating the z-transform of a sequence of N samples." The z-transform is defined as

$$
X(z)=\sum_{n=-\infty}^{\infty} x[n] z^{-n}
$$

And its computation is facilitated by the CZT algorithm. The DFT is a special case of the ztransform and it has some limitations which can be eliminated using the CZT algorithm [14].

The reference [15] shows that the relationship between a continuous frequency response $H$ and its corresponding discrete frequency response $\hat{\mathrm{H}}$ is:

$$
\hat{H}(f)=\left(H(f) \cdot \Pi(f T) \cdot \Delta_{\frac{1}{K T}}(f)\right) * \Delta_{\frac{1}{T}}(f)
$$

Where $\Pi$ is a rectangle function, $\Delta$ is a sampling function, $T$ is the sample period and $\mathrm{K}$ is defined as the integer impulse response sample length. This reference also explores issues with real time domain responses, time aliasing, causality, interpolation and re-sampling of discrete frequency data, which are useful to perform the signal processing properly.

Finally, with the $\mathrm{H}$ function, the S-parameters can be obtained. If the analysis was made to the reflected signal (TDR), the scattering parameters $S_{11}$ (source in port 1) and $S_{22}$ (source in port 2) can be achieved. $\mathrm{S} 11$ in $\mathrm{dB}$ is given by:

$$
S_{11}=20 \log \left|H_{T D R}(f)\right|
$$

However, if the analysis is made to the transmitted signal (TDT), the scattering parameters $\mathrm{S}_{21}$ (source in port 1) and $\mathrm{S}_{12}$ (source in port 2) can be achieved. $\mathrm{S}_{21}$ in $\mathrm{dB}$ is given by:

$$
S_{21}=20 \log \left|H_{T D T}(f)\right|
$$




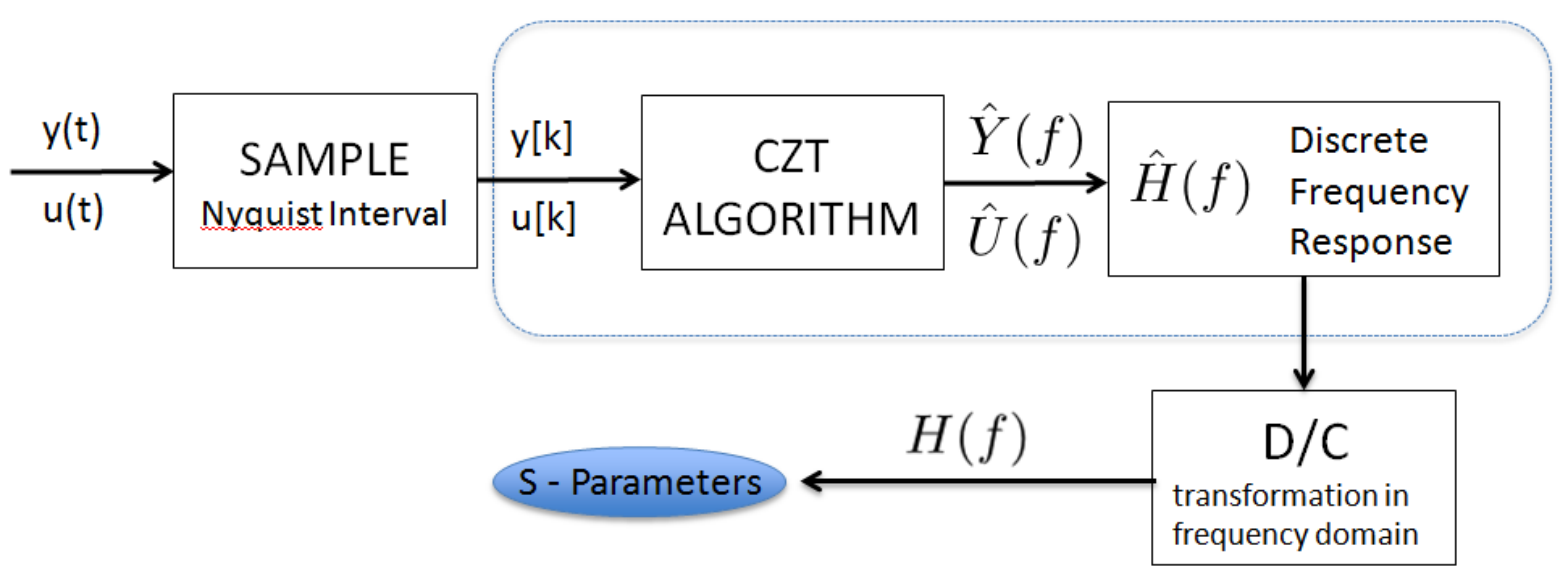

Fig. 2-(a). Block diagram of the signal processing steps.

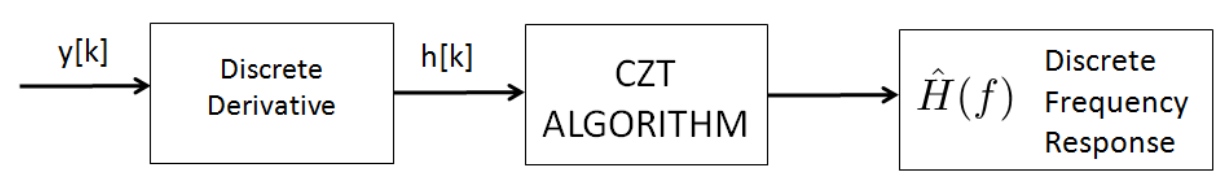

Fig. 2-(b). Alternate implementation of the dashed blue area.

Figure 2-(a) shows a block diagram representation of the signal processing that has been discussed, while figure 2-(b) shows an alternative implementation for the dashed blue area; note that this alternative is only possible if the input signal is a step function, due to the linearity of the differentiation operator. This alternative implementation is simpler, as there is no requirement for the input signal to be measured, stored and processed, lowering computational costs.

\section{C. $\quad$ Filtering Noisy Data}

Let denote the discrete signal obtained either from TDR or TDT as $\mathrm{y}$, thus $\mathrm{y}_{\mathrm{k}}$ represents the $\mathrm{k}^{\text {th }}$ position of vector $\mathrm{y}$. Since we are supposing that the data is noisy, $\mathrm{y}$ is a sum of the theoretical signal $\mathrm{w}$ and a error $\epsilon$ that we assume Gaussian [16]. In other words $y=w+\epsilon$. The Hodrick Prescott filter is defined as follows:

$$
w=\underset{w}{\operatorname{argmin}}\left(\sum_{k=1}^{K}\left(w_{k}-y_{k}\right)^{2}+\lambda \sum_{k=2}^{K-1}\left[\left(w_{k+1}-w_{k}\right)-\left(w_{k}-w_{k-1}\right)\right]^{2}\right)
$$

The first sum minimizes the error, which means that the output of the filter must keep similarity with the noisy signal. The second sum is used for minimizing the second difference of the output signal, thus minimizing the variation of the signal's derivative. For the second sum, $\lambda$ is a parameter that controls how fine the smoothing will be. The resulting signal is a smoother approximation of the original noisy data.

\section{EXPERIMENTAL RESULTS}

Using a pulse generator, a step signal is generated. Before the device under test (DUT) can be analysed, this signal is routed directly to the time domain oscilloscope's input port through a coaxial 
copper cable, as shown in figure 3 . This is the signal that will act as the input for the DUT, as discussed in the previous section, and is shown on figure 4.

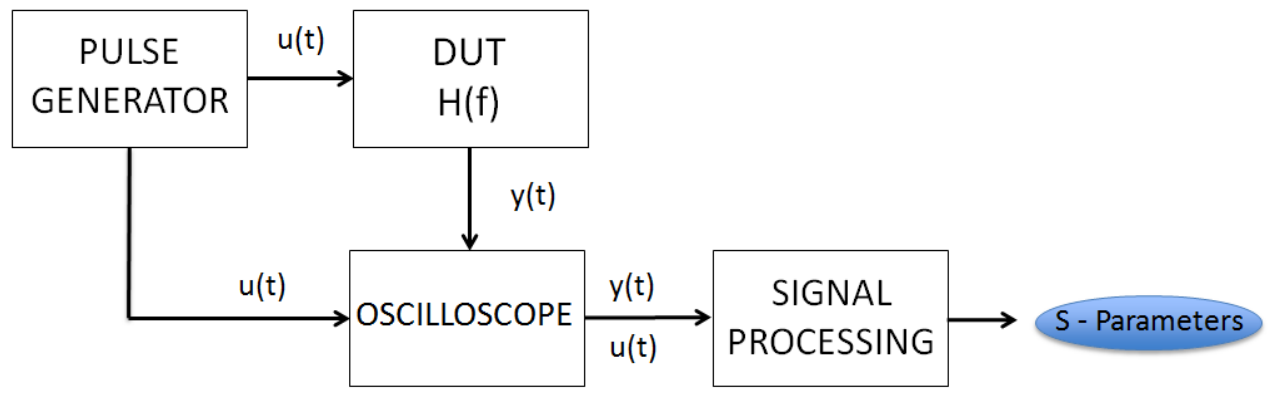

Fig. 3-(a). Block diagram of the experimental setup.

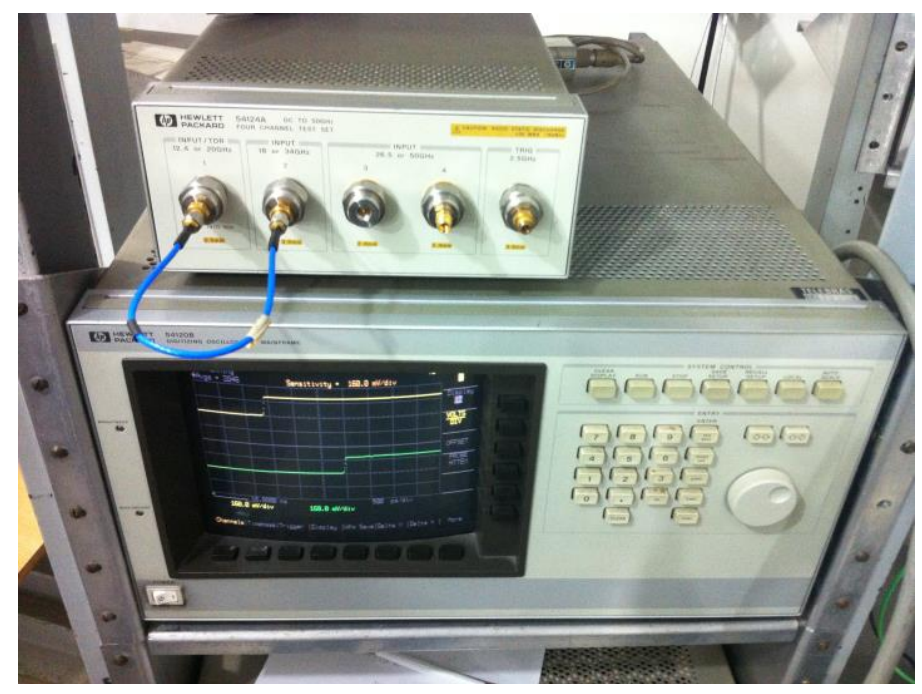

Fig. 3-(b). Experimental setup.

The time domain response data from the coaxial cable can be saved for later processing, and the DUT is added to the network. As discussed in the previous section, the step signal will create reflected and transmitted pulses upon interacting with the DUT, and both of those pulses are recorded by the time domain oscilloscope, with their data saved to a computer.

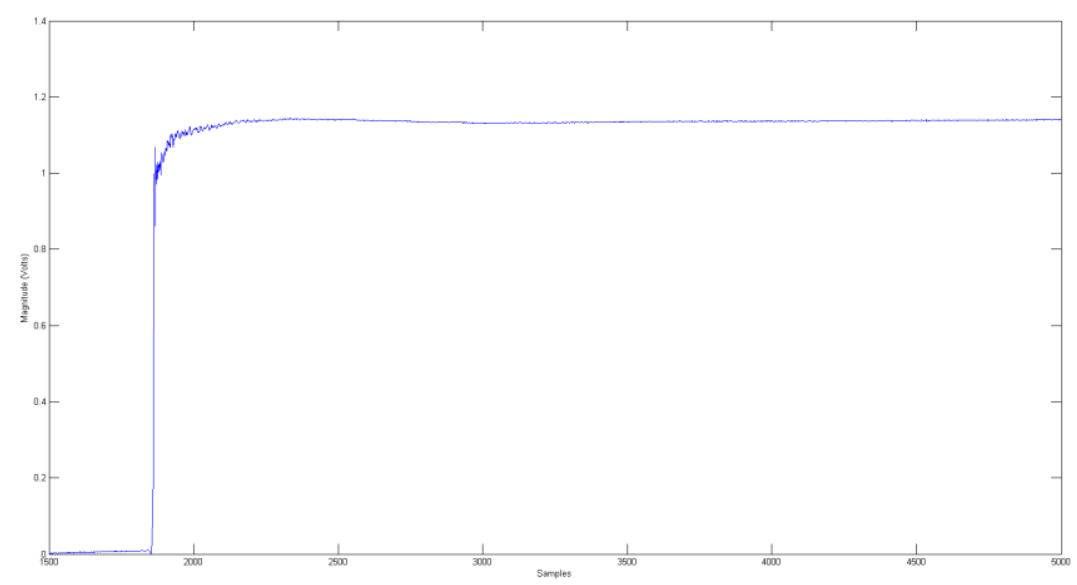

Fig. 4. Smooth time domain input data. 
To implement the alternative shown on Figure 2-(b), the source signal must be a step signal so that differentiating the time response of the DUT results in the impulse response of the system. In addition, the rise time of the step must be short enough that the step can be considered ideal; for comparison purposes, Figure 5 shows the frequency spectrum of the derivative of the response signal, which must be close to the ideal case for the method to be valid, that is, a spectral band that is flat for all frequencies. Since the step signal is not ideal, a high frequency decay can be seen on Figure 5, but the smoothness of the response justifies the alternative implementation of figure 2-(b), as the result is very close to ideal.

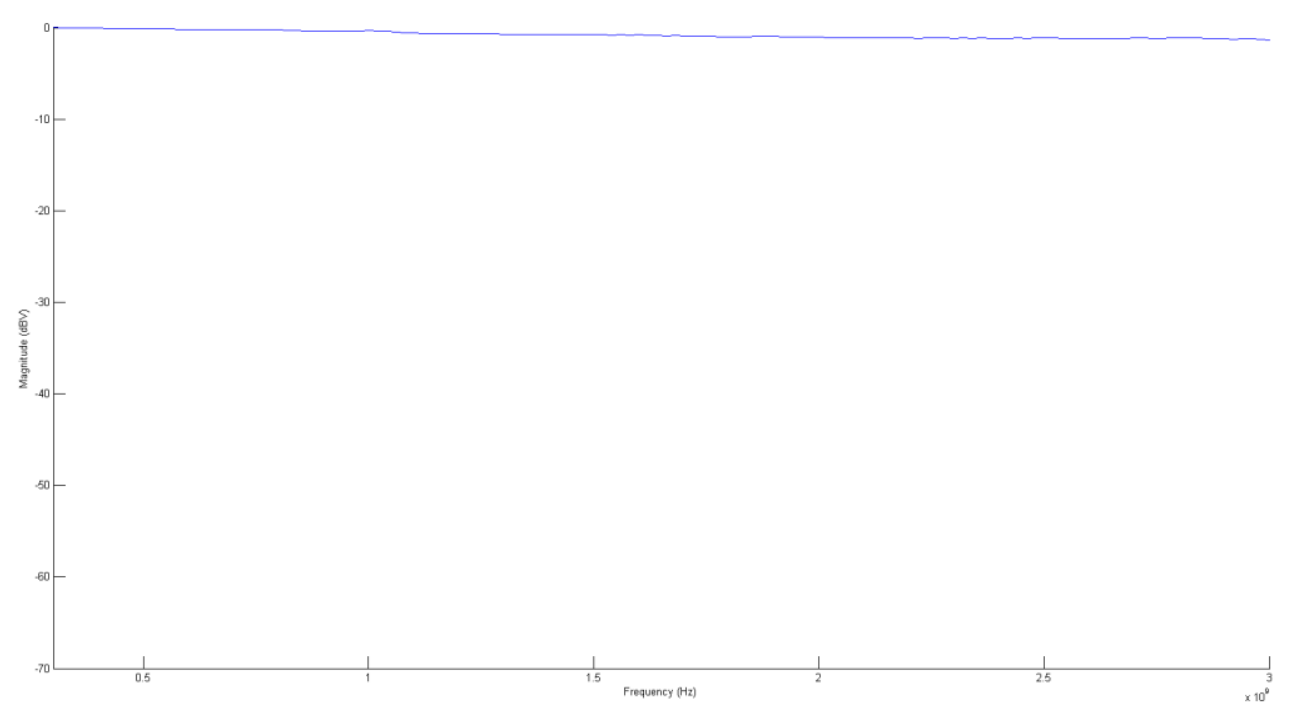

Fig. 5. Frequency domain information for the derivative of the input signal.

Tests were made with two bandpass filters, with central frequencies $925 \mathrm{MHz}$ and $1065 \mathrm{MHz}$, shown in figure 6. Time domain data was collected and processed with MATLAB software, and then compared to data from a network analyser.

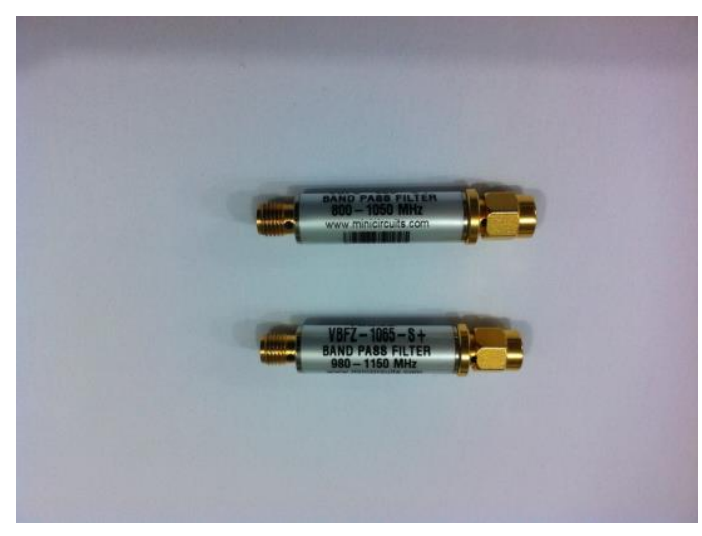

Fig. 6. $925 \mathrm{MHz}$ and $1065 \mathrm{MHz}$ filters used for testing.

The time domain data from the response of the DUT is sent to the frequency domain through the Fourier Transform discussed previously. This is the output signal of the system. The smoothed time domain response from each filter tested is shown in figures 7 and 8. 


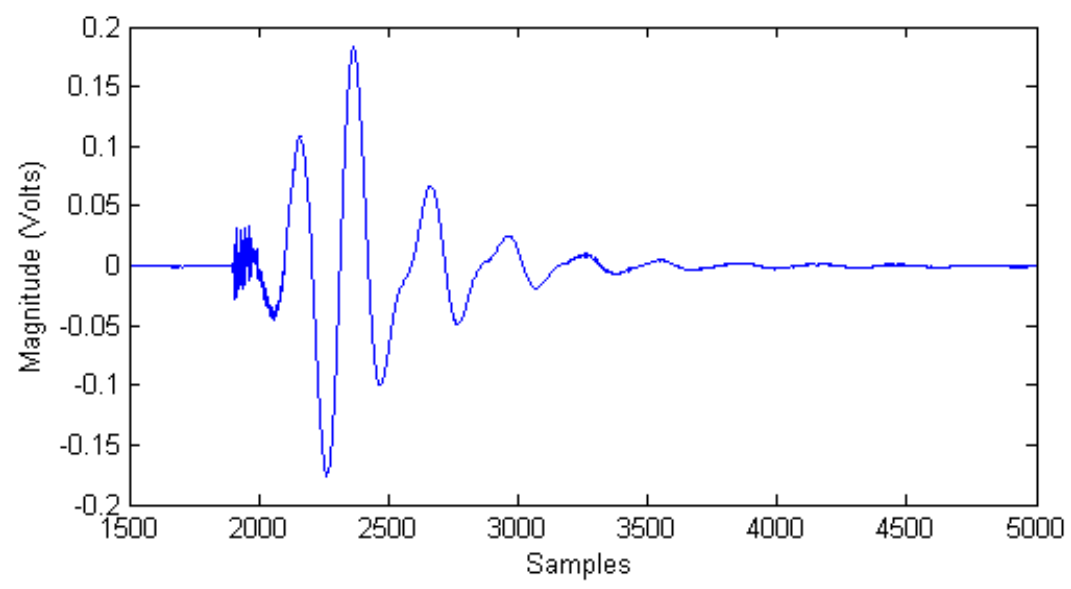

Fig. 7. Smooth time domain output data for the $925 \mathrm{MHz}$ filter.

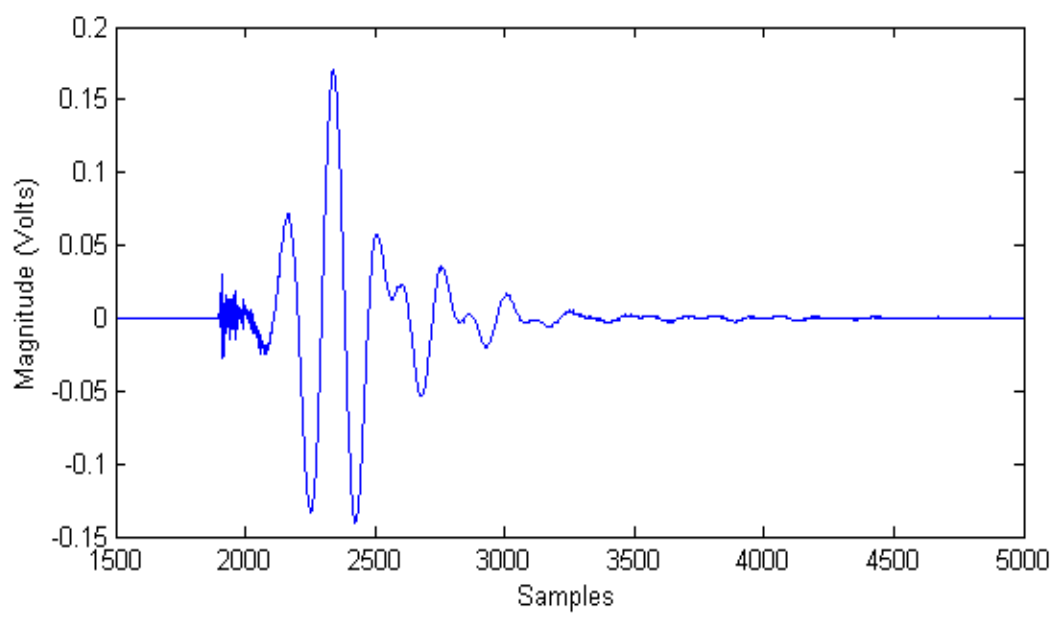

Fig. 8. Smooth time domain output data for the $1065 \mathrm{MHz}$ filter.

The transfer function of the system can now be calculated. With the transfer function, the S-

Parameters can be calculated as discussed in the previous section. Finally, figures 9 and 10 show the frequency response data for both filters in black, as well as the smoothed result in red and the results from a standard network analyser model in green, for comparison.

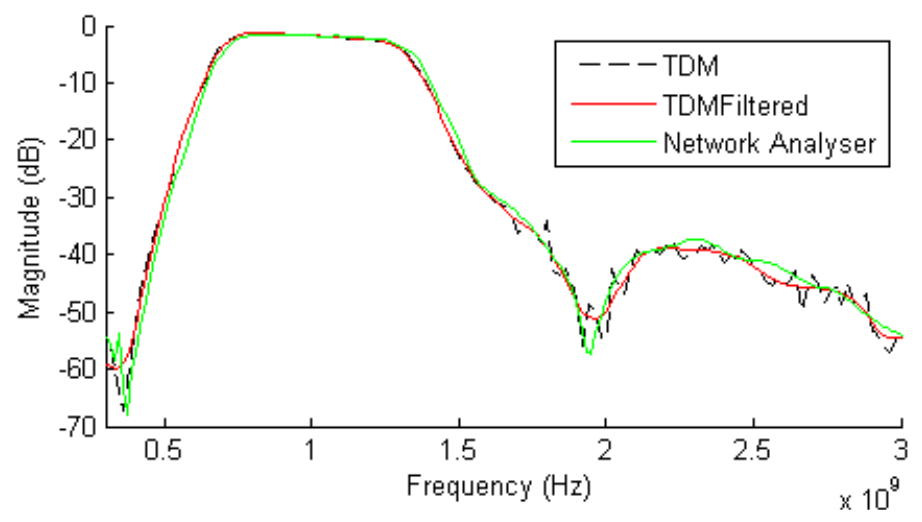

Fig. 9. Transmission Results for $925 \mathrm{MHz}$ filter. The green line shows the result from a vector network analyser, the dashed black line shows the result of the processing technique and the red line shows the result of the processing technique after mathematical filtering was performed. 


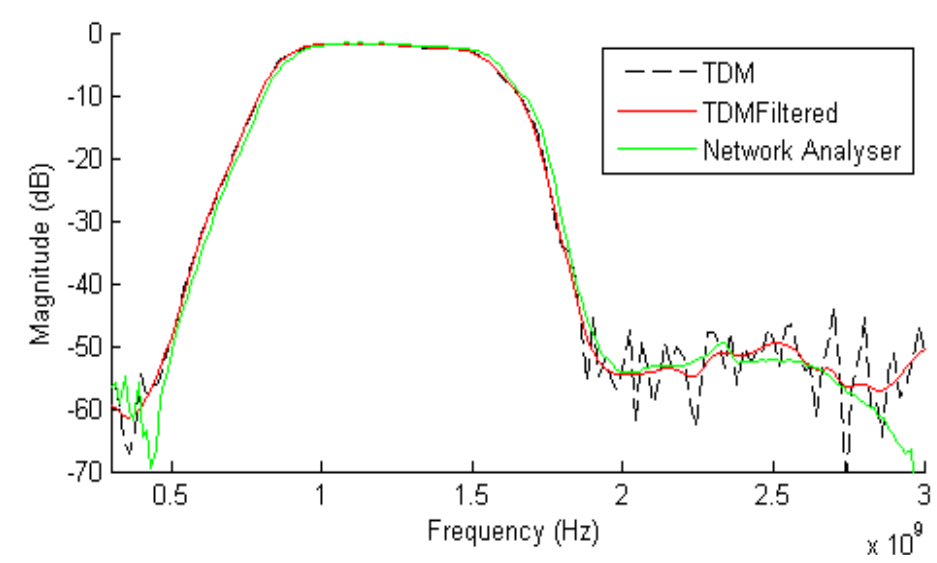

Fig. 10. Transmission Results for 1065MHz filter. The green line shows the result from a vector network analyser, the dashed black line shows the result of the processing technique and the red line shows the result of the processing technique after mathematical filtering was performed.

Despite being close to ideal, the high-frequency decay in the derivative of the input signal shows that there will be an increasing error in the output data at high frequencies: as it decays, the frequency response rises due to equation 7 . Another factor that can be seen from the unsmoothed dashed black line is that the results become increasingly noisy at very low magnitudes (below $-50 \mathrm{~dB}$ ) due to the computations reaching the noise floor of the DFT algorithm utilized; this can be seen more easily on the $1065 \mathrm{MHz}$ filter result, as it reaches lower magnitudes.

\section{DISCUSSION}

The results from the time domain analysis match with the results from the network analyser for the entire operational band of both filters tested. It was observed that the noise in the time domain data is an important factor in obtaining good results, and that time domain responses have a trend of being extremely noisy at their start.

The precision for the pulse's rise time of the equipment used for the time domain data acquisition is also an important factor to consider. With a more precise and faster equipment, not only the frequency band for which the technique matches increases, but noise and errors are also reduced in magnitude.

The results shown in this paper are for TDT analysis only, that is, for the $S_{21}$ and $S_{12}$ parameters. The technique is also applicable to TDR data and can also be employed for phase analysis of signals, but concerns of noise and precision appear, as both reflection waves and phase detection are more sensible to these errors.

In a future analysis, both TDR and phase information could be obtained by the use of advanced, more precise, faster and less noisy equipment.

\section{REFERENCES}

[1] K. Kurokawa, "Power waves and the scattering matrix," Microwave Theory and Techniques, IEEE Transactions on, vol. 13, no. 2, pp. 194_202,1965.

[2] S. Pannala, Development of time domain characterization methods for packaging structures. $\mathrm{PhD}$ thesis, Georgia Institute of Technology, 1999. 
[3] D. C. DeGroot and R. B. Marks, "Optimizing time-domain network analysis" in ARFTG Conference Digest-Fall, 46th, vol. 28, pp. 19_28,IEEE, 1995.

[4] Agilent Application Note, Time Domain Reflectometry Theory, 2013.

[5] S.-J. Kim, K. Koh, S. Boyd, and D. Gorinevsky, "11 trend filtering,” Siam Review, vol. 51, no. 2, pp. 339-360, 2009.

[6] A. Savitzky and M. J. Golay "Smoothing and diferentiation of data by simplified least squares procedures" Analytical chemistry, vol. 36,no. 8, pp. 1627-1639, 1964.

[7] R. J. Hodrick and E. C. Prescott, "Postwar us business cycles: an empirical investigation, " Journal of Money, credit, and Banking, pp. 1-16,1997.

[8] P. H. Eilers, ”A perfect smoother, ” Analytical chemistry, vol. 75, no. 14,pp. 3631-3636, 2003.

[9] D. Gorinevsky, "Optimal estimate of monotonic trend with sparse jumps, ” in American Control Conference, 2007. ACC'07, pp. 1051-[ 1056, IEEE, 2007.

[10] S. M. Wentworth, Fundamentals of electromagnetics with engineering applications. Wiley, 2004.

[11] C. E. Shannon, "Communication in the presence of noise, " Proceedings of the IRE, vol. 37, no. 1, pp. 10-21, 1949.

[12] A. V. Oppenheim, A. S. Willsky, and S. H. Nawab, Signals and systems, vol. 2. Prentice-Hall Englewood Cliffs, NJ, 1983.

[13] K. Runtz and D. Hack,"A multistage dft-fft-czt approach for accurate e-cient analysis of sparsely distributed spectra," in Electrical and Computer Engineering, 2002. IEEE CCECE 2002. Canadian Conference on", vol. 1, pp. 127-132, IEEE, 2002.

[14] L. Rabiner, R. W. Schafer, and C. M. Rader, ” The chirp z-transform algorithm” Audio and Electroacoustics, IEEE Transactions on, vol. 17, no. 2, pp. 86-92, 1969.

[15] P. J. Pupalaikis, "The relationship between discrete-frequency s-parameters and continuous-frequency responses, ” 2012.

[16] S. Haykin, Communication systems. John Wiley \& Sons, 2008. 